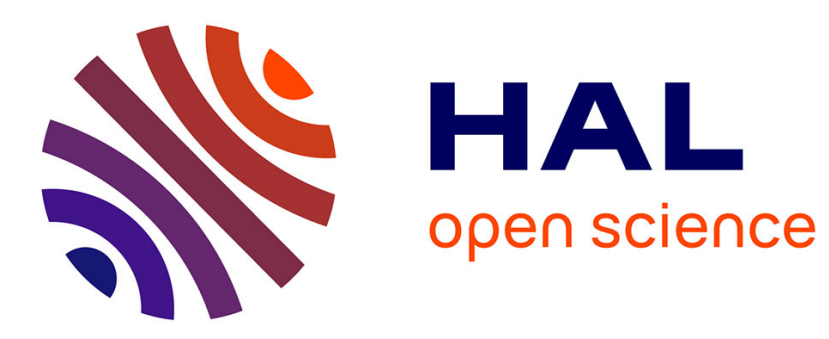

\title{
Dynamics of molecular transport by surfactants in emulsions
}

Yousr Skhiri, Philipp Gruner, Benoît Semin, Quentin Brosseau, Deniz Pekin, Linas Mazutis, Victoire Goust, Felix Kleinschmidt, Abdeslam El Harrak, J. Brian Hutchison, et al.

\section{To cite this version:}

Yousr Skhiri, Philipp Gruner, Benoît Semin, Quentin Brosseau, Deniz Pekin, et al.. Dynamics of molecular transport by surfactants in emulsions. Soft Matter, 2012, 8 (41), pp.10618. 10.1039/C2SM25934F . hal-02148774

\section{HAL Id: hal-02148774 https://hal.science/hal-02148774}

Submitted on 26 Mar 2021

HAL is a multi-disciplinary open access archive for the deposit and dissemination of scientific research documents, whether they are published or not. The documents may come from teaching and research institutions in France or abroad, or from public or private research centers.
L'archive ouverte pluridisciplinaire HAL, est destinée au dépôt et à la diffusion de documents scientifiques de niveau recherche, publiés ou non, émanant des établissements d'enseignement et de recherche français ou étrangers, des laboratoires publics ou privés. 


\title{
Dynamics of molecular transport by surfactants in
}

\section{emulsions}

\author{
Yousr Skhiri $^{a *}$, Philipp Gruner ${ }^{b *},{\text { Benoît } \operatorname{Semin}^{b} \text {, Quentin Brosseau }}^{b}$, \\ Deniz Pekin $^{a}$, Linas Mazutis $^{c}$, Victoire Goust $^{a}$, Felix Kleinschmidt ${ }^{a}$, \\ Abdeslam El Harrak ${ }^{a, d}$, J. Brian Hutchison ${ }^{e},{\text { Estelle } \text { Mayot }^{a},}$ \\ Jean-François Bartolo ${ }^{a}$, Andrew D. Griffiths ${ }^{a}$, Valérie Taly ${ }^{a, f}$ \\ and Jean-Christophe Baret $^{b}$
}

\begin{abstract}
${ }^{a}$ Institut de Science et d'Ingénierie Supramoléculaires (ISIS), Université de Strasbourg, CNRS UMR 7006, Strasbourg (France). ${ }^{b}$ Max-Planck Institute for Dynamics and Self-organization (MPIDS), 37077 Göttingen (Germany), ${ }^{c}$ Vilnius University Institute of Biotechnology, Vilnius LT-02241 (Lithuania) and Harvard University, SEAS, 9 Oxford Street, Cambridge MA (USA) ${ }^{d}$ Raindance Technologies France, Strasbourg (France), ${ }^{e}$ Raindance Technologies, Inc., Lexington, MA (USA), ${ }^{f}$ Universit Paris Sorbonne Cit; INSERM UMR-S775, 75270 Paris Cedex (France)

* These two authors contributed equally to the work
\end{abstract}

This document is the last author version of the manuscript published as: Dynamics of molecular transport by surfactants in emulsions, Y. Skhiri et al., Soft Matter (2012)

https://doi.org/10.1039/C2SM25934F 


\begin{abstract}
We consider the dynamics of equilibration of the chemical potential of a fluorophore in a monodisperse emulsion containing droplets with two initially different concentrations of the fluorophore. Although the exchange mechanism involves a single time-scale at the droplet (microscopic) level, the organisation of the droplets determines the exchange dynamics at the population (macroscopic) level. The micelle concentration in the continuous phase and the chemistry of the fluorophore control the microscopic exchange rate while the disorder of the initial condition determines the power-law of the long-time scale, recovered in a minimal analytical model. We also show here that an additive in the droplet such as Bovine Serum Albumine (BSA) acts on the microscopic exchange rate and slows down the exchange process, by increasing the solubility of the fluorophore in the dispersed phase rather than creating a viscoelastic layer at the droplet interface.
\end{abstract}

\title{
Introduction
}

Droplet-based microfluidics has proven a very effective tool for the miniaturisation and automation of biological assays, for single cell analysis, DNA screening or drug screening ${ }^{1-3}$. In order to reliably function, the droplets have to fulfill three major conditions: they must be stable against coalescence, biocompatible, and their components must remain encapsulated over time, three properties controlled by the surfactant molecules ( $\mathrm{see}^{4,5}$ and reference therein). Understanding the transport of molecules between dispersed microcontainers is not only beneficial for the optimization of microreactors for biochemical applications ${ }^{1}$, but is also of relevance to other encapsulation systems

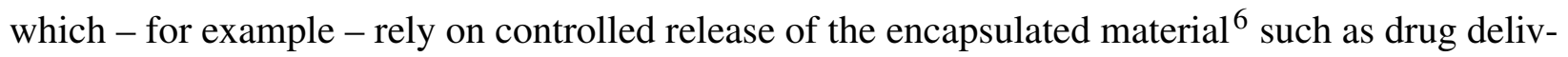
ery systems ${ }^{7}$, for applications such as emulsion polymerisation, or for cell physics ${ }^{8}$. The stability of droplets is an old problem linked to the ageing of emulsions ${ }^{9,10}$. In the case of a cream, a mayonnaise or a pharmaceutical formulation, all droplets of the sample contain the same material and ageing is linked to the transport of the dispersed phase through the continuous phase or through the bilayers that can form between droplets ${ }^{11}$. With the recent applications of droplet emulsions 
for biotechnological applications, the paradigm for emulsion ageing has slightly shifted. In the case of storage of a chemical compound library or a library of single genes in droplets, all droplets contain different molecules, possibly at different concentrations ${ }^{12-16}$. It becomes then important to understand and control the ageing of such emulsions by the exchange of solute.

The fine control of droplet actuation and of droplet size distribution, and the accessibility of short timescales $(\sim \mathrm{ms})$ in microfluidic emulsification - difficult to achieve in bulk-emulsification - makes it appealing as a new tool to quantitatively study the physics and physical chemistry of interfaces and emulsions ${ }^{17-23}$. Studies on solute exchange have already been performed in bulk emulsions ${ }^{9,24-26}$, but these studies do not capture the microscopic details of the exchange at the single droplet or interface level. Such insights are now accessible by the microfluidic control of droplets $11,21,27$, but the understanding of collective dynamics in population of droplets is still lacking $^{28}$.

In the present manuscript, we address the dynamics of molecular exchange between droplets in a mixed emulsion. We measure experimentally the relaxation of concentration differences in an emulsion (produced in microfluidics) initially containing droplets with two different concentrations of a fluorescent dye and make the link between the microscopic exchange between two adjacent droplets and the macroscopic kinetics (at the scale of the emulsion). We study the case of water in fluorinated oil inverse emulsion as they represent the most promising systems for biotechnology applications ${ }^{5}$. Fluorinated oils are used to circumvent the exchange of molecules between droplets since organic compounds are highly insoluble in these oils ${ }^{29}$ : the chemical stability of encapsulation is improved compared to mineral or organic oils.

\section{Materials and methods}

\section{Chemicals}

Resorufin sodium salt, fluorescein sodium salt, rhodamine 101 inner salt, sulforhodamine 101 were purchased from Sigma-Aldrich. Solution were prepared by dilution in Phosphate Buffer Saline 
(PBS) 1x, obtained by a 10 fold dilution in millipore water of a PBS solution (10x, Roth). Bovine Serum Albumine (BSA) was purchased from Acros Organics (Albumine bovine, fraction V).

\section{Surfactant}

We used droplets of water in a fluorinated oil (HFE-7500, 3M) stabilised against coalescence by a block-copolymer surfactant, see Figure 1(a). The surfactant was prepared from the commercially available carboxylic acid Krytox 157-FSH (Dupont) and Jeffamine polyetheramines (ED 600, Huntsmann). We used surfactant solution prepared from two different batches and refered in the following as KryJeffa ${ }^{(1)}$ and $\operatorname{KryJeffa}^{(2)}$. The synthesis scheme was based on the synthesis described in Holtze et al. ${ }^{30}$.

\section{Surfactant characterizations}

To characterize our system, the surface tension at different surfactant concentrations were measured for the KryJeffa - HFE mixtures using pendant droplet method (DataPhysics OCA). Equilibria are reached after a time of the order of several minutes to several hours, depending on surfactant concentration for droplets of volumes of $\sim 1$ to $20 \mu \mathrm{L}$. Using Dynamics Light Scattering (Malvern Zetasizer), we measured the scattered intensity of the surfactant solution as a function of concentration. We used the automatized optimised mode for signal acquisition and rescaled the data by the corresponding gain provided by the manufacturer. From the scattered intensity, we deduced the Critical Micellar Concentration (CMC) of the surfactant. The size of the scattering objects was obtained by the time-correlation of the scattered signal. The two methods lead to a value of CMC of the surfactant in HFE-7500 in the range 0.02-0.04\% (Figure 1(b,c)). The DLS measurements also showed an increase of the scattering signal corresponding to an increase of the number of objects with the surfactant concentration (quasi-linear relationship) with a weak change in the size of the reverse micelles at higher concentrations (from a radius of $80 \mathrm{~nm}$ to $120 \mathrm{~nm}$ over 3 decades in concentration). 


\section{Microfabrication}

Microchannels of depth $60 \mu \mathrm{m}$ were produced with standard soft-lithography techniques of replica molding of a SU-8 master in Polydimethylsiloxane ${ }^{31}$. The PDMS stamp was then bound to glass

after oxygen plasma activation and hydrophobized using a commercial coating agent (Aquapel, PPG Industries). A collection vial was prepared using a $1 \mathrm{~mL}$ plastic syringe (BBraun) cut at both ends with a scalpel. Two cylindrical PDMS blocks cut from a crosslinked PDMS slab were prepared: in one PDMS cylinder, one hole was punched with a Harris Unicore biopsy punch (0.75 mm diameter) to allow a connection with a teflon tubing. Two holes were punched in the second cylinder. Both cylinders were then used to close both ends of the vial.

\section{Chip connection}

All flow rates were controlled by Nemesys syringe pumps (Cetoni GmbH). The bottom of the collection vial was connected to the outlet of the droplet production device at one hole and to a syringe at the second one. The syringe was actuated by the syringe pumps to either push or withdraw liquid (Control Pump). The top of the collection vial was connected back to the chip. In a preliminary phase the collection vial was filled with oil-surfactant solution (Figure 2 (a)).

\section{Droplet production, collection and reinjection}

Droplet-based microfluidic systems were used to produce monodisperse emulsion. Droplets of resorufin in PBS solutions $(0 \mu \mathrm{M}$ and $10 \mu \mathrm{M})$ were produced in parallel at two different nozzles by flow focusing in a fluorinated oil (HFE-7500, 3M). The droplets contained a fluorescein concentration used to encode the droplet type $(150 \mathrm{nM}$ and $50 \mathrm{nM})$. The measurement of the fluorescein fluorescence provided a way to determine if droplets initially contained $0 \mu \mathrm{M}$ or $10 \mu \mathrm{M}$ resorufin, even after the resorufin has equilibrated and the concentration in all droplets is identical. The oil flow rate was $100 \mu \mathrm{L} / \mathrm{min}$ (except when BSA is used, the oil flow rate being increased to $200 \mu \mathrm{L} / \mathrm{min}$ ), and each acqueous flow rate $50 \mu \mathrm{L} / \mathrm{min}$ corresponding to droplet volume of $0.3 \mathrm{~nL}$. 
Droplets were stabilised against coalescence by the surfactant at concentrations ranging from $0.1 \%$ (limit of emulsion stability) to 5\% (w/w). No coalescence was observed on chip. As demonstrated in a previous study ${ }^{32}$, preventing droplets from touching each other after generation increases emulsion stability. Therefore we designed the channels with an incubation line of about 1 centimeter to increase droplet stability. During the stabilization time of the whole system, the Control Pump CP (Figure 2(b)) was set up to 'infuse' and the droplet flow towards the outlet. When the system was stabilised, CP was set to 'withdraw " with a flow rate tuned to stop the flow at the outlet of the collection vial. The emulsion was collected in the vial. After a few minutes (typically 5 minutes), a volume of about 500 microlitres of emulsion was stored in the vial ( $\sim 10^{6}$ droplets). The production of the droplets was stopped, CP was set to 'infuse' (typically at $1 \mu \mathrm{L} / \mathrm{min}$ ). The droplets were then flowing out of the collection vial towards the reinjection part of the chip where the fluorescence readout took place (typically at 10 - 100 droplets per second). The delay time between the first droplet was produced and the first droplet was measured is typically 5 minutes.

\section{Fluorescence measurement}

The fluorescence signal of each droplet was measured as it was flowing through the microfluidic channel using a laser-induced fluorescence setup equipped with a $473 \mathrm{~nm}$ and a $532 \mathrm{~nm}$ laser (DragonLaser) and photomultiplier tube (PMT, Hammamatsu) in a system similar to the one described in Baret et al. ${ }^{12}$ (Figure 2 (b,c)). The laser spot size was a few microns in diameter, much smaller than both the droplet and the channels (typically 100 microns). The optical filters and dichroics were purchased from AHF, the mounts and optical accessories from Thorlabs. Figure 3(a) shows a typical time trace of the droplets as they flow through the laser. For each droplet, we recorded the maximum fluorescence intensity in the green (fluorescein signal) and orange (resorufin signal) colors. We define the relative fluorescence unit as the measured voltage (U, from the PMT) rescaled by the Gain $G$ as: $\mathrm{RFU}=U / G^{6.8}$. The exponent 6.8 depends on the PMT type, has been checked experimentally and corresponds to the data of the manufacturer. We recorded in realtime the droplet intensity as a function of time using a FPGA board (cRIO, National Instruments) 
controlled by a home-made Labview routine. The measurement of the maximum of the signal is optimized for fast processing with our FPGA board (up to several thousands of droplets per second processed in real time) but is sometimes sensitive to noise levels and laser instabilities leading to small variations in the measurements (of maximum 10 percent). The single point measurement provided a tool to measure the kinetics of equilibration of the emulsion.

\section{Data processing}

Data were post-processed with home-made scripts using standard toolbox in Matlab. From the raw data, we generated the time distribution of the fluorescence by binning typically 2000 droplets (Figure 3(b)). We generated color plots corresponding to the time evolution of the histogram (Figure 3(c)). From the signals, we first analysed the green fluorescence to distinguish the two populations of droplets. We checked that fluorescein signals were constant at the time-scale of our experiments. The orange fluorescence of both population was then fitted by Gaussian distributions to obtain the means $m_{1}$ and $m_{2}$ of each droplet population. In the linear regime of fluorescence (as it is the case here), the fluorescence was proportional to dye concentration. Therefore the value $m_{2}-m_{1}$ is proportional to the difference of concentration of the dye. By rescaling the data by the initial value, we obtained a measurement of the concentration difference rescaled by the initial concentration: $\Delta c / c_{0}=\left(m_{2}-m_{1}\right) /\left(m_{2}-m_{1}\right)_{0}$. This parameter - equal to 1 at the beginning of the experiment and to 0 when the concentrations are equilibrated - provided means to compare experiments performed for different dyes and different initial ratio of dye concentrations.

\section{Experimental results}

We performed the exchange experiments with resorufin. Two populations of monodisperse droplets $(0.3 \mathrm{~nL})$ with different concentration of resorufin encoded by fluorescein are mixed. We measured the kinetics of equilibration of the chemical potential of the resorufin by fluorescence. Figure 3(b) and (c) show the typical time distribution of the droplet fluorescence over a few hours for $C=0.5$ 
$\%_{w / w}$. Starting from two distinct populations of droplets, the equilibrium was reached after several hours. The average value of the fluorescence signal of the two populations was constant throughout the measurement indicating that the dye is preserved (no chemical modification nor bleaching). This also indicates that the dye mainly remains in the aqueous compartments. We observed that the initial dye concentration did not significantly impact the time-scale of the process which justifies the rescaling of the concentration difference by the initial concentration $c_{0}$ (Figure $4(\mathrm{~b})$ ). In addition, the droplet size was not changing over the time-scale of the experiments and no transport of water was observed between the droplet: the transport of water is prevented by the high concentration of salts in the PBS buffer which was the same in all droplets ${ }^{11,23}$.

We performed the experiments for several surfactant concentrations above the CMC of the surfactant in HFE-7500. Figure 3(d) shows the time variation of the concentration difference over several hours of incubation. The rate of the equilibration increases with the surfactant concentration. All the experimental data collapse on a single master curve (Figure 4(a)) using a single time-scale $\tau_{e}$ for each concentration indicating a similar process for the exchange at all concentrations above the CMC. $\tau_{e}$ is inversely proportional to the surfactant concentration (Figure 4(b)) over almost two orders of magnitude in surfactant concentration. In contrast to other systems where the exchange is linked to bilayer formation ${ }^{11}$, our system shows the importance of the surfactant remaining in the continuous phase on the transport process. We observed no bilayers formed at any of these surfactant concentrations, therefore our results suggest that reverse micelles behave as nanocontainers increasing the solubility of the compound in the continuous phase ${ }^{26}$. The rescaled experimental data fit with an exponential decay only in the early time kinetics (Figure 4(a)). This exponential decay is not maintained at longer time-scale (closer to equilibrium), where a powerlaw behaviour is experimentally observed. In the region where the power-law is obtained, the fluorescence distributions of both population overlap. Notably, we can still measure the mean of each population due to the coding by fluorescein.

We also observed that the chemistry of the molecule influences the rate of exchange. As an example, fluorescein exchange is barely detactable over 24 hours. The exchange rates of sulforho- 
damine and dextran texas red are as well barely measurable on the time-scale of the experiments while rhodamine 101 is fully exchanged within minutes. This confirms previously published results ${ }^{33}$ indicating that addition of sulfonate groups increases the chemical stability of the encapsulation. We also show that the time-scale $\tau_{e}$ depends on the presence of Bovine Serum Albumin (BSA) in the droplet. The decrease of exchange by bovine serum albumin has been previously reported in mineral oils ${ }^{28}$. Here we measured here a 18 fold decrease of the exchange rate of resorufin by adding BSA in the droplet at a concentration of $25 \mathrm{mg} / \mathrm{mL}$ and the exchange rate is still proportional to the surfactant concentration (Figure 4 (b)).

One hypothesis, proposed previously in mineral oil emulsion, is that BSA acts as a barrier against exchange at the surface of the droplet ${ }^{28}$. We performed the following experiment to test this hypothesis and clarify the role of BSA in the control of exchange. All droplets contained the same resorufin concentration but the BSA $(25 \mathrm{mg} / \mathrm{mL})$ was present in only one type of droplet (Figure $4(\mathrm{c}, \mathrm{d})$ ), those droplets being encoded with a $150 \mathrm{nM}$ fluorescein code (droplet containing no BSA also contain $50 \mathrm{nM}$ fluorescein). We measured the variation of the resorufin fluorescence signal as a function of time. In this experiment, we also observed the exchange of resorufin leading to an a priori counter-intuitive result: the difference in fluorescence is increasing during incubation (with a time-scale of order 20 minutes). At equilibrium, the fluorescence signals of the two types of droplets are different by a factor of 15 (Figure 4 (d)) and the most fluorescent droplets are those containing the BSA. The concentration of resorufin in the droplets containing BSA is 15 fold larger than in droplets containing no BSA. Our experimental results clearly contradicts the assumption that BSA creates a thin viscoelastic layer at the interface of the droplets acting as a kinetic barrier. If the BSA only acts as a barrier to exchange, the chemical potential of the resorufin in the droplets being initially at equilibrium, the fluorescence signal should remain constant. The time-scale of the exchange process is similar to the time-scale of exchange previously measured for the corresponding surfactant concentration (2\%) which indicates that the mechanism of transport by surfactant is not affected by the presence of BSA. Finally, the ratio between the equilibrium fluorescence of the two droplet populations matches quantitatively the decrease of exchange rate 
obtained in Figure 4 (b). In this particular case, BSA is efficiently used to extract and concentrate resorufin.

\section{Modelling}

The experimental results shown in figure 4(a) display a decay slower than exponential. For the quantity of interest, i.e. the difference between the mean values of the two subpopulations $\Delta c / c_{0}$, the system dimension affects the late kinetics with the power law $\Delta c / c_{0} \sim t^{-3 / 2}$ (Figure 4(a)). We developed a model to describe the exchange and explain the collective dynamics in the emulsion.

We consider now an ensemble of droplets separated by a porous membrane of thickness $L^{34}$. For simplicity, let us consider droplets arranged on the nodes of a three dimensionnal square lattice. The droplet on the node $(k, l, m)$ contains molecules in a solvent at concentration $c_{k, l, m}$ and exchanges molecules with its 6 first neighbours only (4 neighbours in 2 dimensions, as displayed in Figure 5, 2 neighbours in 1 dimension). The area of contact between the compartments is $s$ (a fraction of the droplet surface area $S$ ) and the volume of the compartment is $V$ (Figure 5). The difference in resorufin chemical potential between the two compartments is responsible for the molecular flow with a flux $J$ along each axis proportional to the concentration difference between neighbours $\delta c$ as described for membranes or bilayers ${ }^{11,35,36}$ :

$$
J=-P \delta c
$$

$J$ is expressed in $\mathrm{mol} / \mathrm{s} / \mathrm{m}^{2}$, the concentrations in $\mathrm{mol} / \mathrm{m}^{3}$ and the permeability $P$ has the dimension of a speed $(\mathrm{m} / \mathrm{s})$. In this case the mass balance and Fick's law leads to a discrete version of the heat equation:

$$
\frac{\partial c}{\partial \tilde{t}}=\underline{\Delta} c
$$

where $\underline{\Delta}$ is a discrete Laplacian and $\tilde{t}=t / \tau$ is the dimensionless time with $\tau=V /(P s)$. For periodic 
boundary conditions, the eigenvalues of the discrete Laplace operator are in three dimensions:

$$
\lambda_{p, q, r}=-4 \times\left[\sin ^{2}\left(\pi \frac{p}{N_{x}}\right)+\sin ^{2}\left(\pi \frac{q}{N_{y}}\right)+\sin ^{2}\left(\pi \frac{r}{N_{z}}\right)\right]
$$

(for $0 \leq p, q, r<N-1$ ) and the eigenvectors

$$
V_{p, q, r}(k, l, m)=\omega_{p}^{k} \omega_{q}^{l} \omega_{r}^{m}
$$

with

$$
\omega_{p}=\exp \left(2 i \pi p / N_{x}\right)
$$

Due to the special form of the eigenvectors, the time evolution of the ensemble of $N$ droplets is analytically expressed in the Fourier space as:

$$
\mathscr{F}(c)=\mathscr{F}\left(c_{i}\right) \mathrm{e}^{\lambda_{p, q, r} \tilde{t}}
$$

where $c_{i}$ is the initial distribution and $\mathscr{F}$ denotes the Discrete Fourier Transform. Since Eq. 1 is linear, we subtract in the following the mean value of concentration and normalize them to 1 to represent concentrations between -1 and 1 , defining two subpopulations of droplets.

The smallest eigenvalue of the Laplace operator is -12 (in three dimensions) corresponding to the fastest decaying mode. The smallest eigenvalue is -8 in two dimensions, -4 for one dimension and -2 for a single droplet pair. It corresponds in all dimensions to an eigenmode of perfectly alternating array, where an empty droplet is surrounded solely by filled droplets and vice versa. An alternated three dimensional packing would equilibrate following an exponential decay with a time scale $\tau / 12$. All other configurations decay more slowly.

The relaxation kinetics towards a uniform concentration depends on the excitation of the eigenmodes in the initial conditions. Experimentally, the mixing of droplets in the incubation vial leads to a random distribution of droplet positions. To investigate the effect of the disorder, we solved Eq. 1 for a completely random and uncorrelated initial condition. The scaling law can then be 
recovered analytically. Since the value of the initial concentrations is 1 or -1 depending on the subpopulation, $\Delta c / c_{0}$ is proportional to the scalar product of $c$ by $c_{i}$. Using Parseval's theorem, this scalar product can be computed in the Fourier space. In 3-d, we have:

$$
\frac{\Delta c}{c_{0}} \propto \sum_{p, q, r}\left|\mathscr{F}\left(n_{0}\right)\right|^{2} \mathrm{e}^{\lambda_{p, q, r} \tilde{t}}
$$

Due to the completely random initial condition, all the modes are statistically excited with the same weight (which is an unusual initial condition) and $\Delta c / c_{0} \propto \sum_{p, q, r} \mathrm{e}^{\lambda_{p, q, r}, \tilde{t}}$. At long time, only the modes with the smaller eigenvalues remain, so that

$$
\lambda_{p, q, r} \sim-4 \pi^{2}\left(\left(p / N_{x}\right)^{2}+\left(q / N_{y}\right)^{2}+\left(r / N_{z}\right)^{2}\right)
$$

and the sum can be extended to $+\infty$. For large systems, we can use a continuum approximation, and

$$
\frac{\Delta c}{c_{0}} \sim \int_{0}^{+\infty} \int_{0}^{+\infty} \int_{0}^{+\infty} \mathrm{e}^{-4\left[\left(\pi p / N_{x}\right)^{2}+\left(\pi q / N_{y}\right)^{2}+\left(\pi r / N_{z}\right)^{2}\right] \tilde{t}} \mathrm{~d} p \mathrm{~d} q \mathrm{~d} r
$$

which scales as $t^{-3 / 2}$ (in 3d). Figure 5(e) shows a summary of the model data in the three dimensional case and the corresponding asymptotic regimes. Our model can also be solved in 1-d and 2-d cases and for any set of initial conditions using similar schemes. Similarly, we also recover analytically that $\Delta c / c_{0}$ scales in 1-d like as $\tilde{t}^{-1 / 2}$, and in 2 -d as $\tilde{t}^{-1}$.

At short-times, an argument similar to the one at large time-scales leads to $\Delta c / c_{0} \sim \sum_{p, q, r} \mathrm{e}^{\lambda_{p, q, r} \tilde{t}}$. Expanding the exponential close to 0 leads to $\Delta c / c_{0} \sim 1-\lambda \tilde{t}$, where $\lambda$ is the average value of the eigenvalues, which is equal to -6 in three dimensions (-4 in two dimensions and -2 in one dimension). Therefore the early time kinetics in a random initial distribution is an exponential decay two times slower than the exponential decay of the fastest mode (the alternating case) of the corresponding dimension.

In summary, the relaxation of the chemical potential at the emulsion level is a function of the initial distribution of the droplet in the packing. The maximum rate is obtained for an alternating 
packing and corresponds to the rate of exchange at the droplet pair level (corrected by the number of nearest neighbours). The early time kinetics of a random distribution is twice slower but has the same order of magnitude, while alternatively, the rate of exchange of an emulsion made of two layers would equilibrate with a much longer time-scale, that can be several orders of magnitude longer. These results are generic and do not rely on the exact description of the exchange mechanism at the droplet level.

\section{Discussion}

Our experiments show how the packing and the random organisation of the droplets affect the kinetics of equilibration of the emulsion. A single time-scale controls the exchange and we measured how this time-scale is influenced by surfactant concentration, the chemistry of the molecules to be transported and presence of additives such as BSA in the dispersed phase. We want here to relate our experiments to models of membrane permeability ${ }^{23,35,36}$.

When the rate-limiting step is diffusion in the membrane (case I of Zwolinksi et al. ${ }^{35}$ ), the membrane permeability is expressed as $P=K D / L^{35}, K$ being the partition coefficient of the dye between the reservoir and the membrane, $D$ the diffusion coefficient of the dye and $L$ the membrane thickness. One of the control parameter is therefore the partition coefficient between the compartment and the membrane. Non-fluorinated organic molecules, such as resorufin, are typically highly insoluble in fluorinated oils similar to those used for the continuous phase in this study ${ }^{29}$. We have not been able to directly measure the partition coefficient between the aqueous phase and the oil, or the oil-surfactant mixtures as, (i) the optical properties of the dye seem to differ in water and in oil and (ii) the presence of surfactant makes such bulk measurement technically challenging. Furthermore, performing an experiment with BSA in half of the droplets provided a means to indirectly address the role of the partition coefficient. Starting from droplets which all have an identical concentration of resorufin, but where half of the droplets contained $25 \mathrm{mg} / \mathrm{mL}$ of BSA, the concentration of resorufin in the two populations diverged until, at equilibrium, there was 
15 fold higher concentration of resorufin in the droplets containing BSA than in those containing no BSA. Therefore adding BSA provides a means to modify the parameter $K$ without modifying the dye or the continuous phase. Exchange experiments performed with $25 \mathrm{mg} / \mathrm{mL}$ BSA in all droplets and with resorufin in only half led to a decrease of exchange rate by a factor of about 18 which matches quantitatively the corresponding decrease of $K$. Our experiments are therefore consistent with a diffusion-limited permeability model. This mechanism is also consistent with the results obtained with other dyes showing a decrease of exchange when sulfonated groups are added $^{33}$ : if the solubility in the aqueous phase increases, the rate of exchange decreases.

The decrease of solute exchange mediated by BSA has here been quantitatively measured with fluorinated oils, but was previously reported for non fluorinated oils ${ }^{28}$. One of the initial explanation for the effect of BSA is the creation of a protein layer at the inner surface of the droplets which provides a barrier in a capsule-like manner as suggested earlier for mineral oils ${ }^{28}$. This layer would decrease the permeability of the medium between the droplets ${ }^{37}$. In this case, the ratelimiting step would be the diffusion through the BSA layer (case II or III of Zwolinksi et al. ${ }^{35}$ ). However with such picture one would not expect that (i) the surfactant concentration plays a role in the presence of BSA and that (ii) starting from equilibrated concentrations, the final state shows a concentration difference. Our experiments show unambiguously that BSA acts on the solubility of the dye (a thermodynamic property) rather than on kinetic parameters such as energy barriers. The quantitative link between the equilibrium concentration ratio of resorufin in aqueous droplet with and witout BSA and the exchange rate with BSA is consistent with a model of porous membranes where the permeability is controlled by partition coefficient between the aqueous phase (the droplets) and the continuous phase (fluorinated oil containing surfactant). We speculate that the change in partition coefficient of resorufin caused by BSA may be due to the remarkable capacity of serum albumins to bind a structurally diverse set of small molecules, including many drugs $^{38}$. The binding of resorufin, or other solutes, to BSA could potentially increase solubility in the aquous phase and/or decrease solubility in the oil/surfactant phase.

Recently the permeability of a bilayer membrane has been linked to its fluidity ${ }^{11}$. In our 
case, the surfactant does not form bilayers and the micelle concentration in the oil phase plays a significant role in the transport, the exchange rate being proportionnal to micelle concentration. It indirectly shows that the solubility in the continuous phase is increasing linearly with surfactant concentration. Therefore the likely mechanism for transport is through the solubilisation of the dye molecule into the reverse micelles (above the $\mathrm{CMC}$, the free surfactant concentration is constant) which can act as relatively large $(\sim 100 \mathrm{~nm}$ diameter $)$ carrier of molecules between droplets. The shape and size of the micelles could also be an important control parameter in the transport and further experiments investigating the structure of these micelles would be of interest.

Finally, for a given emulsification system, the time-scale $\tau=V /(P s)$ controls the exchange, with a prefactor corresponding to the number of nearest neighbours. We anticipate that the area controlling the transport is proportional to the droplet area $R^{2}$. However, in the case of bilayers, there is no reason to assume that $P$ also scales with the droplet size. Therefore $\tau$ probably has a linear dependence with the droplet size $\tau \sim R / P$ and we expect that a decrease of droplet size will increase the rate of exchange. On the contrary, when no bilayers are formed $P$ integrates a geometric parameter such as the droplet-droplet distance and the scaling will be different. Further experiments investigating these scalings will be of interest. The link between the exchange rates in the different dimensions is determined by the physical details of the membrane between droplets. Microfluidic experiments provide a means to control droplets to study isolated pairs or generate controlled packing in $1 \mathrm{~d}$ or $2 \mathrm{~d}$ and $3 \mathrm{~d}$. By measuring the exchange kinetics in the different dimensions and varying the number of nearest neighbours, it will be possible to extract microscopic information on the membrane (for example what is the exact description of $P$ and $s$ ).

Microfluidic systems have a huge potential to address the remaining open questions by the possible on-chip control of packing geometries, droplet spacing or sizes for a deeper understanding of such transport phenomenae. We plan as a next step to study the effect of the packing geometry on the dynamics of the relaxation as well as the effect of targeted or random droplet rearrangements: Inducing re-ordering of droplets while diffusion occurs might lead to interesting kinetics - especially when the droplet reshuffling can be controlled ${ }^{39}$. Microfluidics will provide, in the 
future, a very powerful platform for the study of such transport problems by the possible control of droplet packing and lateral ordering of the droplets ${ }^{39-41}$. Finally, the mechanisms involved in this study are generic (a diffusive process) and could potentially be applied to a better control of collective effects in droplet population, for example, in active emulsions ${ }^{42-44}$, to control signaling in cell populations (chemotaxis, quorum sensing) entrapped in different ecological niche (here the droplets), or to design new systems for screening compounds by affinity. The ability to control the transport by additives selectively encapsulated in specific droplets in the emulsion would also open the door to applications for separation.

\section{Conclusions}

In summary, microfluidics provides the tools to produce calibrated emulsions and to analyse millions of droplets at very high-throughput. We have measured experimentally the equilibration of the chemical potential of chemicals in an emulsion initially composed of two sub-populations of different dye concentrations. We measured the exchange timescale for a three dimensional packing of randomly mixed droplets. We observed that the exchange timescale in a random packing is controlled by the number of reverse micelles in the continuous phase and relates to a classical description of porous membranes through partition coefficient between the dispersed and continuous phase. We showed that - due to the disorder in the initial condition - the macroscopic kinetics are not a simple exponential decay but a power-law and that the initial conditions have a major influence on the behavior of the system. We also showed how the initial distribution of droplets can affect the time-scale of the exchange. The early time kinetics in a mixed emulsion is only twice slower than the maximum rate, obtained for a perfectly organized alternating packing. Most of the important physics of the relaxation is contained in our simple model: the equilibration process is a classical diffusion process through a Laplace equation from which emerge a dimension-dependent power-law. The transport - as a passive process - leads to equilibration of concentration and can be modulated by additives such as BSA. Selective transport is obtained by controlling the BSA en- 
capsulation in some droplets to concentrate the compound of interest, leading to a versatile control of chemicals in emulsions.

\section{Aknowledgments}

The authors acknowledge the financial support of Region Alsace, of the 'Fondation d'Entreprise EADS' and of the European Commission Framework Program 6 (MiFem). Financial support from the Max-Planck Gesellschaft and from the Deutsche Forschunggemeinschaft through the Sonderforschungbereich 755 (Nanoscale Photonic Imaging) are also acknowledged. The authors are grateful to C. Rick, C. Ben Salem and Z. El Harrak for help and assistance as well as B. Maury, S. Herminghaus, J. Bibette and N. Bremond for helpful discussions.

\section{References}

[1] A. B. Theberge, F. Courtois, Y. Schaerli, M. Fischlechner, C. Abell, F. Hollfelder and W. T. S. Huck, Angew. Chem., Int. Ed., 2010, 49, 5846-5868.

[2] M. T. Guo, A. Rotem, J. A. Heyman and D. A. Weitz, Lab Chip, 2012, 12, 2146-2155.

[3] R. Seemann, M. Brinkmann, T. Pfohl and S. Herminghaus, Reports on Progress in Physics, 2012, 75, 016601.

[4] J. Bibette, F. Leal-Calderon and P. Poulin, Rep. Prog. Phys., 1999, 62, 696-1033.

[5] J.-C. Baret, Lab Chip, 2012, 12, 422-433.

[6] A. Madene, M. Jacquot, J. Scher and S. Desobry, Int. J. Food Sci. Tech., 2006, 41, 1-21.

[7] V. Sadtler, F. Jeanneaux, M. Krafft, J. Rabai and J. Riess, New J. Chem., 1998, 22, 609-613.

[8] J. C. Mathai, S. Mori, B. L. Smith, G. M. Preston, N. Mohandas, M. Collins, P. C. van Zijl, M. L. Zeidel and P. Agre, J Biol Chem, 1996, 271, 1309-1313.

[9] D. McClements and S. R. Dungan, J. Phys. Chem., 1993, 97, 7304-7308.

[10] D. J. McClements, Soft Matter, 2012, 8, 1719. 
[11] A. Thiam, N. Bremond and J. Bibette, Langmuir, 2012, 28, 6291-6298.

[12] J.-C. Baret, Y. Beck, I. Billas-Massobrio, D. Moras and A. D. Griffiths, Chem. Biol., 2010, 17, 528-536.

[13] O. J. Miller, A. E. Harrak, T. Mangeat, J.-C. Baret, L. Frenz, B. E. Debs, E. Mayot, M. L. Samuels, E. K. Rooney, P. Dieu, M. Galvan, D. R. Link and A. D. Griffiths, Proc Natl Acad Sci U S A, 2012, 109, 378-383.

[14] E. Brouzes, M. Medkova, N. Savenelli, D. Marran, M. Twardowski, J. B. Hutchison, J. M. Rothberg, D. R. Link, N. Perrimon and M. L. Samuels, Proc. Natl. Acad. Sci. U. S. A., 2009, 106, 14195-14200.

[15] D. Pekin, Y. Skhiri, J.-C. Baret, D. L. Corre, L. Mazutis, C. B. Salem, F. Millot, A. E. Harrak, J. B. Hutchison, J. W. Larson, D. R. Link, P. Laurent-Puig, A. D. Griffiths and V. Taly, Lab Chip, 2011, 11, 2156 - 2166.

[16] A. B. Theberge, E. Mayot, A. E. Harrak, F. Kleinschmidt, W. T. S. Huck and A. D. Griffiths, Lab Chip, 2012, 12, 1320-1326.

[17] C. Priest, S. Herminghaus and R. Seemann, Appl. Phys. Lett., 2006, 89, 134101-3.

[18] N. Bremond, A. R. Thiam and J. Bibette, Phys. Rev. Lett., 2008, 100, 024501.

[19] J.-C. Baret, F. Kleinschmidt, A. E. Harrak and A. D. Griffiths, Langmuir, 2009, 25, 6088-6093.

[20] A. R. Thiam, N. Bremond and J. Bibette, Phys. Rev. Lett., 2009, 102, 188304.

[21] A. R. Thiam, N. Bremond and J. Bibette, Phys. Rev. Lett., 2011, 107, 068301.

[22] N. Bremond, H. Domejean and J. Bibette, Phys. Rev. Lett., 2011, 214502.

[23] N. Bremond and J. Bibette, Soft Matter, 2012, DOI: 10.1039/c2sm25923k.

[24] P. Fletcher, A. Howe and B. Robinson, J. Chem. Soc., Faraday Trans. 1, 1987, 83, 985-1006.

[25] N. Chidambaram and D. J. Burgess, AAPS PharmSci., 2000, 2, E30.

[26] N. Chidambaram and D. J. Burgess, AAPS PharmSci., 2000, 2, E28.

[27] Y. Bai, X. He, D. Liu, S. N. Patil, D. Bratton, A. Huebner, F. Hollfelder, C. Abell and W. T. S. Huck, Lab Chip, 2010, 10, 1281-1285.

[28] F. Courtois, L. F. Olguin, G. Whyte, A. B. Theberge, W. T. S. Huck, F. Hollfelder and C. Abell, Anal. Chem., 2009, 81, 3008-3016. 
[29] A. Studer, S. Hadida, R. Ferritto, S. Y. Kim, P. Jeger, P. Wipf and D. P. Curran, Science, 1997, $275,823-826$.

[30] C. Holtze, A. C. Rowat, J. J. Agresti, J. B. Hutchison, F. E. Angile, C. H. J. Schmitz, S. Koester, H. Duan, K. J. Humphry, R. A. Scanga, J. S. Johnson, D. Pisignano and D. A. Weitz, Lab Chip, 2008, 8, 1632-1639.

[31] Y. N. Xia and G. M. Whitesides, Annu. Rev. Mater. Sci., 1998, 28, 153-184.

[32] J.-C. Baret, V. Taly, M. Ryckelynck, C. A. Merten and A. D. Griffiths, Med. Sci., 2009, 25, 627-632.

[33] G. Woronoff, A. E. Harrak, E. Mayot, O. Schicke, O. J. Miller, P. Soumillion, A. D. Griffiths and M. Ryckelynck, Anal. Chem., 2011, 83, 2852-2857.

[34] D. Lee, J. N. Ashcraft, E. Verploegen, E. Pashkovski and D. A. Weitz, Langmuir, 2009, 25, 5762-5766.

[35] B. J. Zwolinski, H. Eyring and C. E. Reese, J. Phys. Coll. Chem., 1949, 53, 1426-1453.

[36] S. M. Saparov, Y. N. Antonenko, R. E. Koeppe and P. Pohl, Biophys J, 2000, 79, 2526-2534.

[37] G. Yampolskaya and D. Platikanov, Adv Colloid Interface Sci, 2006, 128-130, 159-183.

[38] P. T., All about albumin: biochemistry, genetics and medical applications, Academic Press, 1995.

[39] E. Surenjav, S. Herminghaus, C. Priest and R. Seemann, Appl. Phys. Lett., 2009, 95, 154104-3.

[40] P. Abbyad, P.-L. Tharaux, J.-L. Martin, C. N. Baroud and A. Alexandrou, Lab Chip, 2010, 19, $2505-2512$.

[41] P. Abbyad, R. Dangla, A. Alexandrou and C. N. Baroud, Lab Chip, 2011, 11, 813-821.

[42] M. Toiya, V. K. Vanag and I. R. Epstein, Angew. Chem., Int. Ed., 2008, 47, 7753-7755.

[43] S. Thutupalli, S. Herminghaus and R. Seemann, Soft Matter, 2011, 7, 1312-1320.

[44] S. Thutupalli, R. Seemann and S. Herminghaus, New J. Phys., 2011, 7, 013021. 
(a)

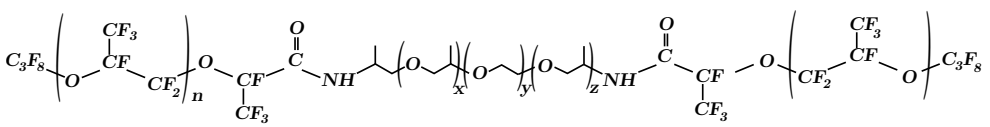

(c)

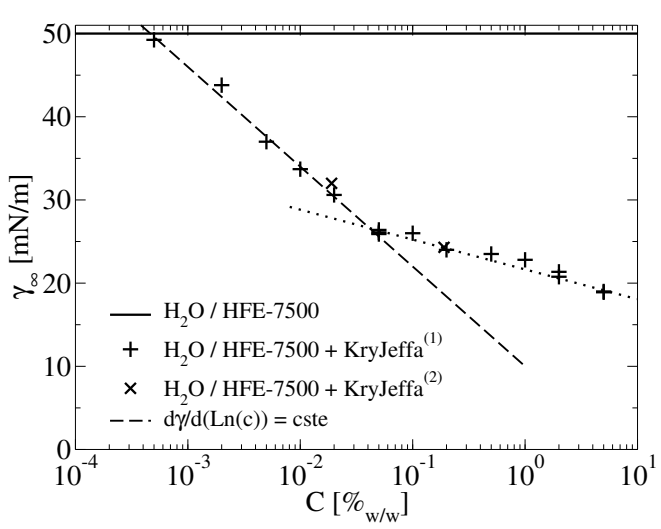

$\left(\mathrm{CF}_{3}\right)_{2} \mathrm{CF}$
$-{ }_{\mathrm{CF}}-\mathrm{C}_{3} \mathrm{~F}_{8}$
1
$\mathrm{O}_{-\mathrm{C}_{2} \mathrm{H}_{5}}$

(b)

(d)

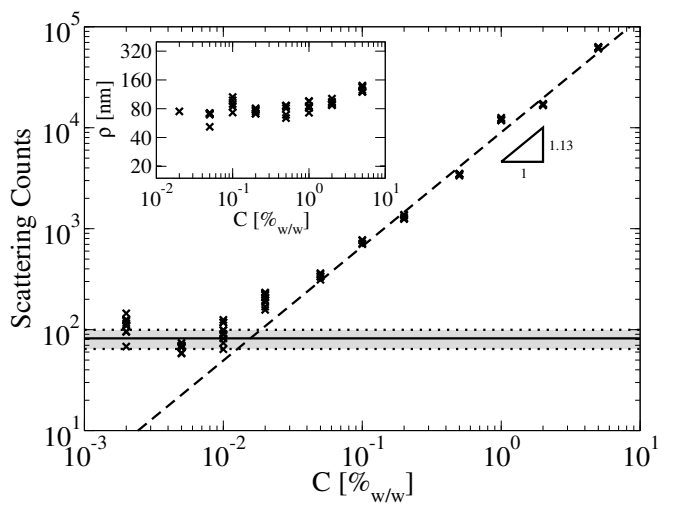

Figure 1 Surfactant solutions (a) The surfactant used in this study is a Krytox-Jeffamine block-copolymer $(x+z \approx 3.6, y \approx 9.0, n=35-40)$ solubilized in fluorinated oil (HFE-7500) (b). The fluorinated oil is used for its low ability to solubilize organic compounds. (c) Equilibrium surface tension for the water/HFE interface in presence of the surfactant at different concentrations. We checked the reproducibility of the synthesis with two batches of surfactant $(+$ and $\times$ ). (d) Dynamic Light Scattering on the oil - surfactant solution. The background count rate of the pure oil is given by the horizontal full line. The grey area represents the standard deviation for 10 measurements. At low surfactant concentrations (below $0.02 \%$, the scattered count rate is in the oil background). Above $0.02 \%$, the count rate of the diffused light is quasi-linear (exponent 1.13). Inset: The radius $\rho$ of reverse micelles measured by correlation of the scattering signal is slightly increasing around $\rho=80 \mathrm{~nm}$ up to $120 \mathrm{~nm}$ at $5 \%$. The first concentration for which a correlation is obtained is $0.02 \%$ although in some runs at $0.02 \%$ the background is too large to obtain a proper correlation. Combining both methods leads to a CMC measurement in the range to 0.02 $0.04 \%_{w / w}$. 


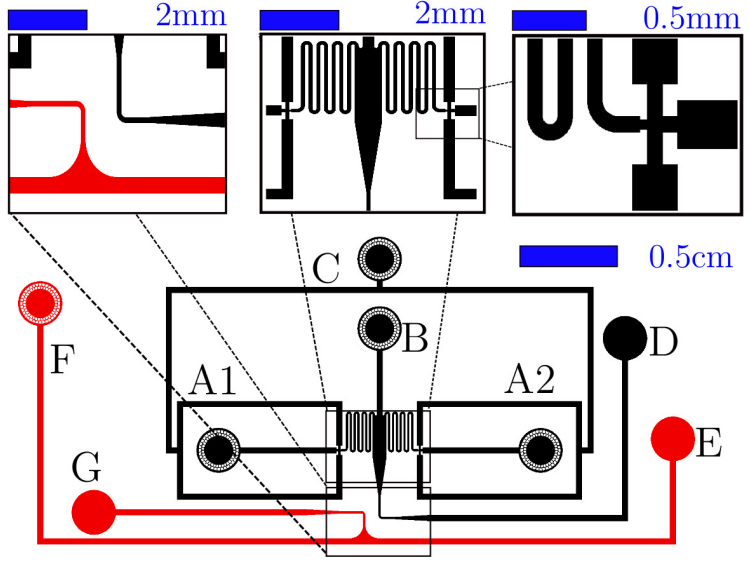

(c)

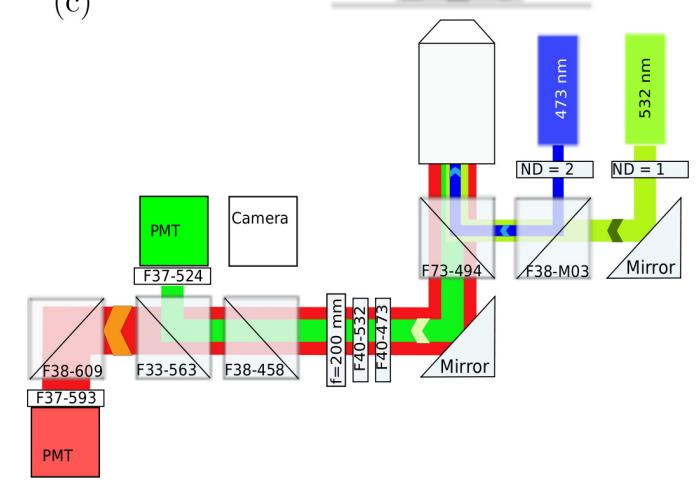

(a)

(b)

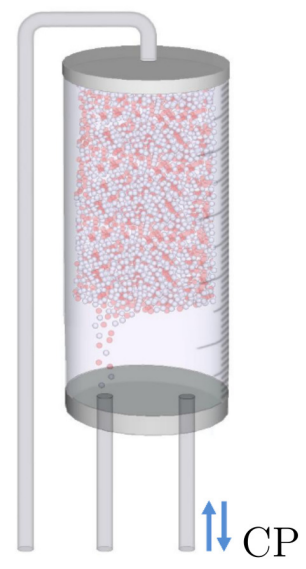

E $\quad \mathrm{D} \quad \mathrm{H}$

(d)

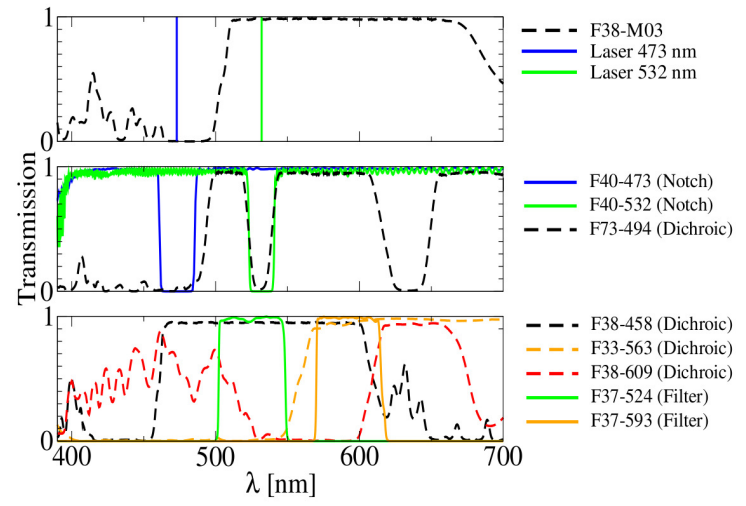

Figure 2 Experimental setup. (a) Sketch of devices for the controlled production of emulsions with two populations of droplets containing two different fluorophore concentrations with zooms on the parts of interest. The chip is made of two parts, the production unit (black) and the reinjection unit (red). In the production unit, the aqueous phase are injected in holes A1 and A2. B is not used here. C is the oil-surfactant inlet. The droplet flow towards the outlet $\mathrm{D}$. At the outlet $\mathrm{D}$, the droplet are collected in the vial (b). The arrows indicate that liquid can be pumped into (reinjection mode) or out (filling mode) of the vial from the Control Pump CP. For reinjection, the droplet flow from the inlet $\mathrm{E}$ to the outlet G. F is an additional oil inlet used to space droplets. (c) Sketch of the laser-induced fluorescence setup for single droplet measurement on 2 colors. (d) Details of the optical components. 
(a)

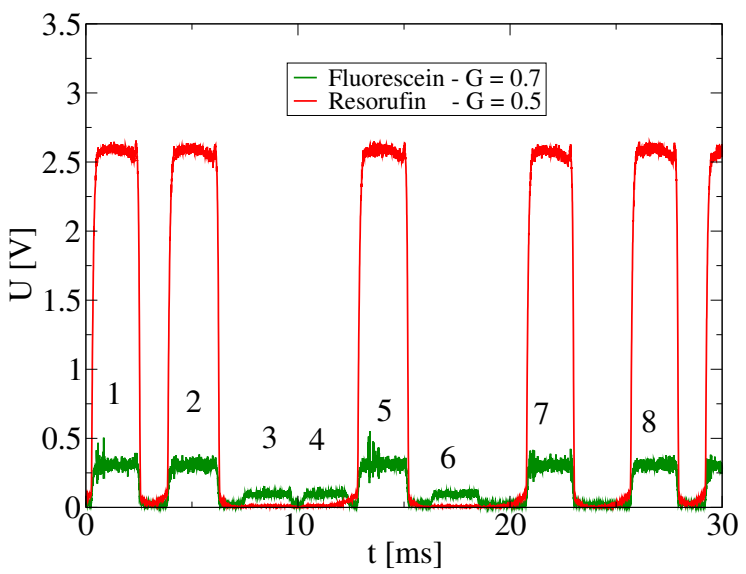

(b)

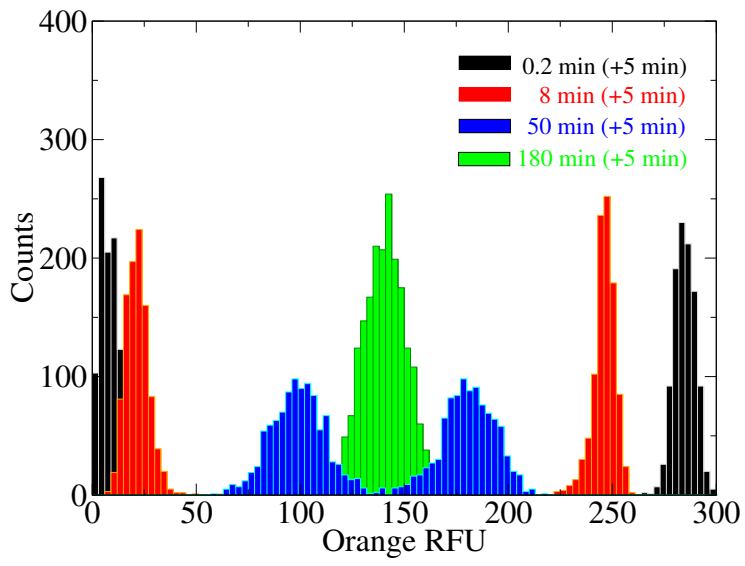

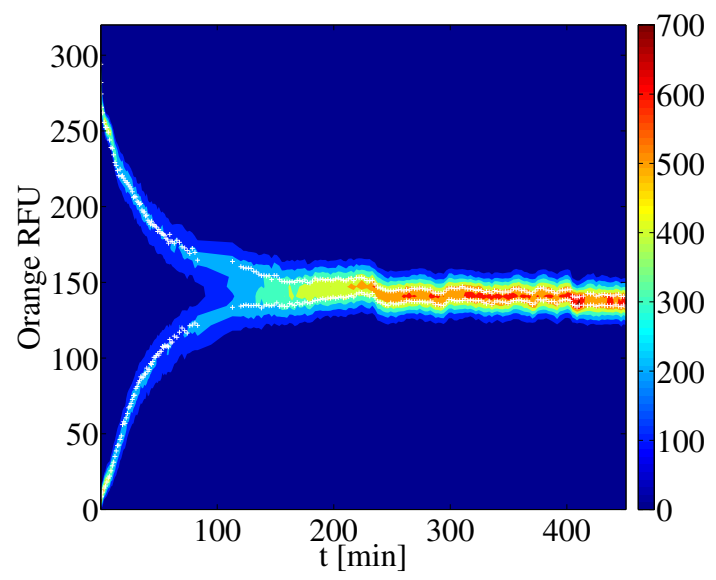

(d)

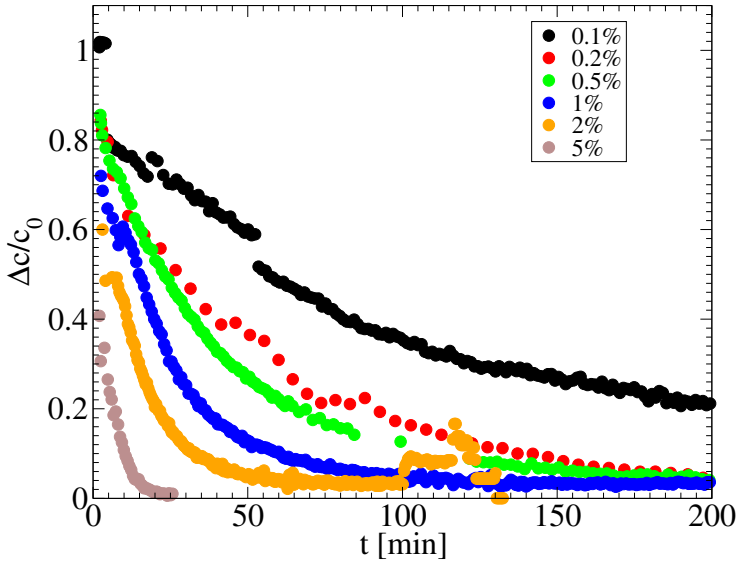

Figure 3 Fluorescence measurement and Analysis (a) Signals measured on the PMT at droplet production. Droplets containing $10 \mu \mathrm{M}$ of resorufin (droplets 1,2,5,7 and 8) are encoded with a high concentration of fluorescein $(150 \mathrm{nM})$. The droplet initially containing no resorufin $(3,4$ and 6$)$ are encoded with a low fluorescein concentration $(50 \mathrm{nM})$. (b) Histogram of resorufin fluorescence at different reinjection time for $C=0.5 \%_{w / w}$ of KryJeffa ${ }^{(1)}$. The histograms are obtained from about 2000 droplets each. (c) Full time histogram (colorplot) of the orange fluorescence recorded over $\sim 8$ hours for a mixed emulsion of resorufin in HFE-7500 with $C=0.5 \%$ w/w of $\mathrm{KryJeffa}^{(1)}$-surfactant showing the equilibration of resorufin chemical potential. (d) Measurement of the concentration difference of the two populations of droplets for increasing surfactant concentration. The kinetics are faster for larger surfactant concentrations. 
(a)

(b)
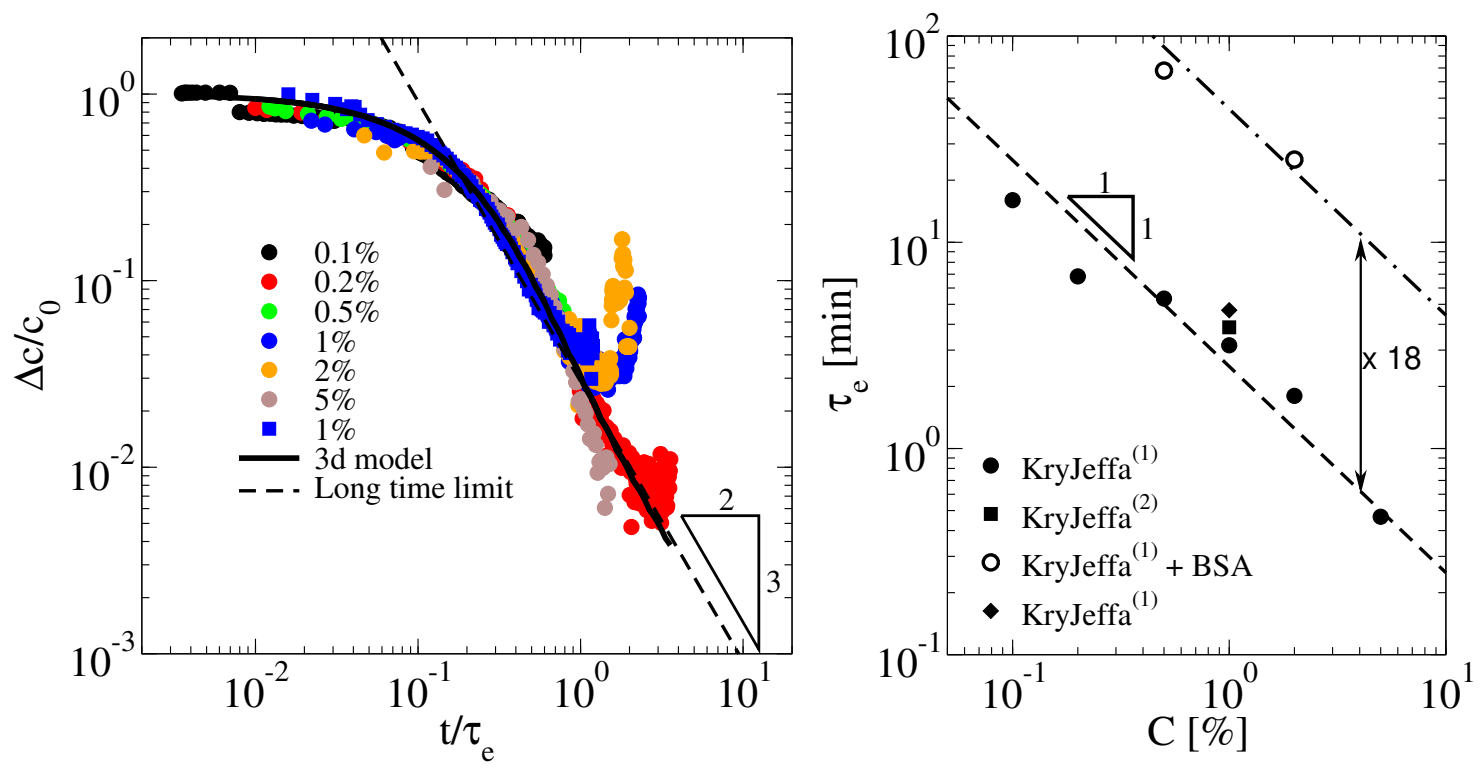

(c)
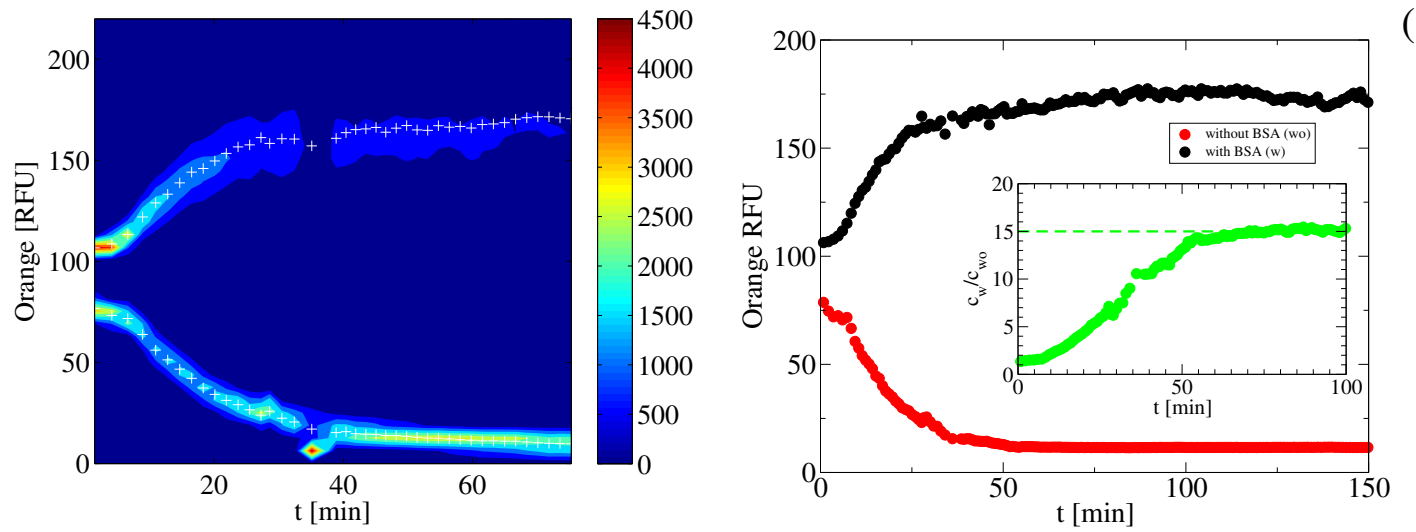

(d)

Figure 4 Rescaling of the exchange kinetics (a) Rescaling of the experimental data by the time-scale $\tau_{e}$ and comparison with our exchange model in the 3-d case with 6 neighbours (full line). At large time, the kinetics follows a power-law with an exponent $-3 / 2$ (dashed line). The increase observed at the end of certain runs are artefacts corresponding to the reinjection of the last droplets from the vial. (b) Time-scale $\tau_{e}$ of the exchange as a function of surfactant concentration $C$ with $10 \mu \mathrm{M}$ initial resorufin concentration. The diamond has been obtained for a $1 \mu \mathrm{M}$ resorufin initial concentration. The open circle correspond to the rescaling in presence of Bovine Serum Albumine (the dashed line and the dotted-dashed line correspond to a linear relationship and the ratio of both is 18). (c) Exchange kinetic at $2 \%$ surfactant concentration when all droplet initially contain $5 \mu \mathrm{M}$ resorufin but only half contain $25 \mathrm{mg} / \mathrm{mL}$ BSA. The equilibrium shows a 15 fold ratio in concentration of resorufin between both types of droplets, $c_{w}$ is the resorufin concentration in the droplet with BSA and $c_{w o}$ without BSA (d). This ratio closely matches the ratio of 18 observed in the exchange rate of (b). 
(e)

(a)

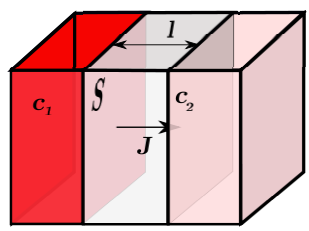

(b)

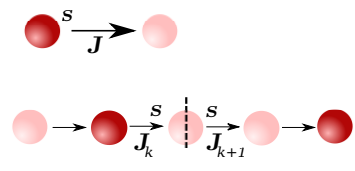

(d)

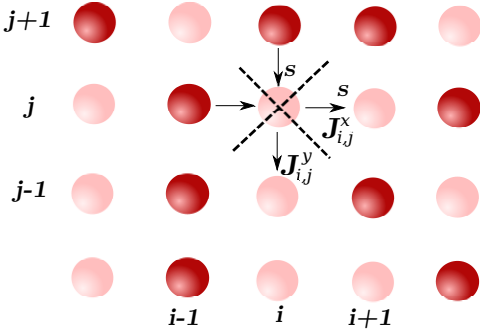

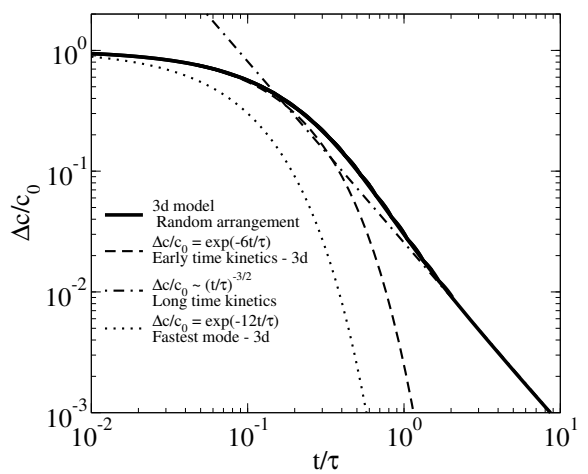

Figure 5 Model for molecular exchange. (a) For a porous membrane separating two compartments, the flux $J$ depends on the concentration difference and geometry parameters $(s, L)$. For droplet pairs we use a similar description (b), the flux being proportional to the concentration difference. In an ensemble of droplets, the exchange occurs with the nearest neighbors: (c) corresponds to a one-dimensional case, (d) the two-dimensional case on a square lattice. (e) The model is solved for a three-dimensional packing on a square lattice. Results of the model and asymptotic regimes in the three-dimensional case. 